\title{
Contents
}

\section{Fractional Zaslavsky and Hénon Discrete Maps}

Vasily E. Tarasov ....................................... 1

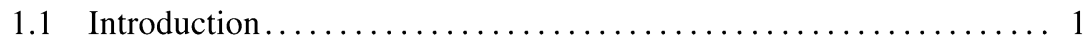

1.2 Fractional derivatives ............................... 3

1.2.1 Fractional Riemann-Liouville derivatives ............... 3

1.2.2 Fractional Caputo derivatives ...................... 4

1.2.3 Fractional Liouville derivatives ................... 6

1.2.4 Interpretation of equations with fractional derivatives ...... 6

1.2.5 Discrete maps with memory $\ldots \ldots \ldots \ldots \ldots \ldots \ldots \ldots \ldots$

1.3 Fractional Zaslavsky maps $\ldots \ldots \ldots \ldots \ldots \ldots \ldots \ldots \ldots \ldots \ldots \ldots$

1.3.1 Discrete Chirikov and Zaslavsky maps ............... 9

1.3.2 Fractional universal and Zaslavsky map ............... 10

1.3.3 Kicked damped rotator map ..................... 12

1.3.4 Fractional Zaslavsky map from fractional differential equations .................................. 13

1.4 Fractional Hénon map .................................. 14

1.4.1 Hénon map................................... 14

1.4.2 Fractional Hénon map ........................... 15

1.5 Fractional derivative in the kicked term and Zaslavsky map ....... 16

1.5.1 Fractional equation and discrete map............... 17

1.5.2 Examples ................................. 20

1.6 Fractional derivative in the kicked damped term and generalizations of Zaslavsky and Hénon maps .............. 21

1.6.1 Fractional equation and discrete map................ 21

1.6.2 Fractional Zaslavsky and Hénon maps ................. 24

1.7 Conclusion ......................................... 25

References....................................... 25

\section{Self-similarity, Stochasticity and Fractionality}

Vladimir V. Uchaikin ..................................... 27 


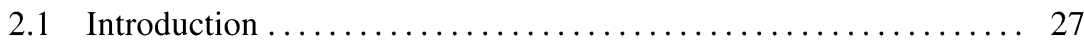

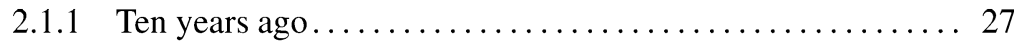

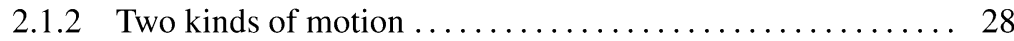

2.1 .3 Dynamic self-similarity ...................... 29

2.1.4 Stochastic self-similarity .................... 30

2.1.5 Self-similarity and stationarity $\ldots \ldots \ldots \ldots \ldots \ldots \ldots \ldots \ldots \ldots$

2.2 From Brownian motion to Lèvy motion ................ 32

2.2.1 Brownian motion ....................... 32

2.2.2 Self-similar Brownian motion in nonstationary nonhomogeneous environment ................ 35

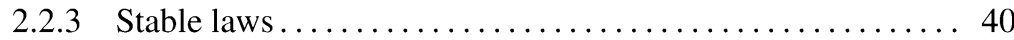

2.2.4 Discrete time Lèvy motion .................. 45

2.2.5 Continuous time Lèvy motion ................ 50

2.2.6 Fractional equations for continuous time Lèvy motion ..... . 51

2.3 Fractional Brownian motion ........................ 54

2.3.1 Differential Brownian motion process ............ 55

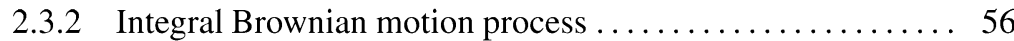

2.3.3 Fractional Brownian motion .................. 58

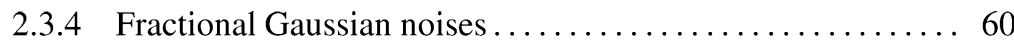

2.3.5 Barnes and Allan model ...................... 61

2.3.6 Fractional Lèvy motion .......................... 62

2.4 Fractional Poisson motion ........................... 64

2.4.1 Renewal processes ......................... 64

2.4 .2 Self-similar renewal processes $\ldots \ldots \ldots \ldots \ldots \ldots \ldots \ldots 65$

2.4.3 Three forms of fractal dust generator ............. 66

$2.4 .4 n$th arrival time distribution $\ldots \ldots \ldots \ldots \ldots \ldots \ldots \ldots \ldots 6 . \ldots \ldots$

2.4.5 Fractional Poisson distribution ..................... 68

2.5 Fractional compound Poisson process $\ldots \ldots \ldots \ldots \ldots \ldots \ldots \ldots$

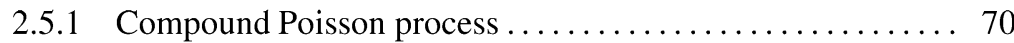

2.5.2 Lèvy-Poisson motion .......................... 71

2.5.3 Fractional compound Poisson motion .............. 73

2.5.4 A link between solutions...................... 74

2.5.5 Fractional generalization of the Lèvy motion ......... 75

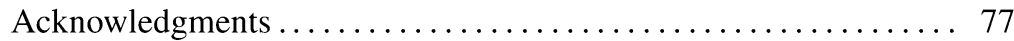

Appendix. Fractional operators . . . . . . . . . . . . . . . . 77

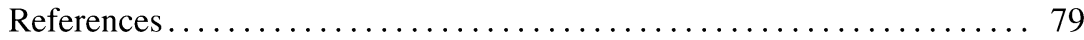

\section{Long-range Interactions and Diluted Networks}

Antonia Ciani, Duccio Fanelli and Stefano Ruffo .............. 83

3.1 Long-range interactions ............................. 84

3.1 .1 Lack of additivity ...................... 85

3.1.2 Equilibrium anomalies: Ensemble inequivalence, negative specific heat and temperature jumps............. 87

3.1.3 Non-equilibrium dynamical properties ............. 92

3.1.4 Quasi Stationary States ..................... 93 
3.1.5 Physical examples ............................. 94

3.1.6 General remarks and outlook ................... 97

3.2 Hamiltonian Mean Field model: equilibrium and out-of-

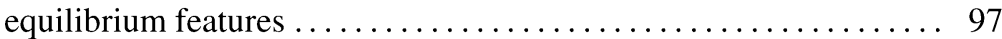

3.2.1 The model .................................. 98

3.2.2 Equilibrium statistical mechanics................. 100

3.2.3 On the emergence of Quasi Stationary States: Nonequilibrium dynamics ....................... 103

3.3 Introducing dilution in the Hamiltonian Mean Field model ...... 120

3.3.1 Hamiltonian Mean Field model on a diluted network ...... 120

3.3.2 On equilibrium solution of diluted Hamiltonian Mean Field .................................. 121

3.3.3 On Quasi Stationary States in presence of dilution........ 123

3.3.4 Phase transition................................... 129

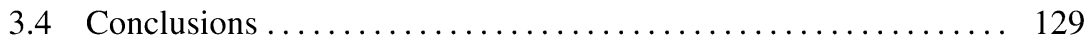

Acknowledgments ................................ 130

References........................................ 130

\section{Metastability and Transients in Brain Dynamics: Problems} and Rigorous Results

Valentin S. Afraimovich, Mehmet K. Muezzinoglu and

Mikhail I. Rabinovich .................................. 133

4.1 Introduction: what we discuss and why now .............. 134

4.1.1 Dynamical modeling of cognition ................... 134

4.1 .2 Brain imaging ............................... 135

4.1.3 Dynamics of emotions ........................ 136

4.2 Mental modes....................................... 137

4.2.1 State space ................................. 137

4.2.2 Functional networks .......................... 137

4.2.3 Emotion-cognition tandem....................... 140

4.2.4 Dynamical model of consciousness .................. 142

4.3 Competition—robustness and sensitivity ................ 144

4.3.1 Transients versus attractors in brain .................. 145

4.3.2 Cognitive variables .............................. 146

4.3.3 Emotional variables ............................ 147

4.3.4 Metastability and dynamical principles.............. 148

4.3.5 Winnerless competition—structural stability of transients .. 148

4.3.6 Examples: competitive dynamics in sensory systems ...... 150

4.3.7 Stable heteroclinic channels ....................... 151

4.4 Basic ecological model ............................... 153

4.4.1 The Lotka-Volterra system ......................... 153

4.4.2 Stress and hysteresis .......................... 155

4.4.3 Mood and cognition............................ 157

4.4.4 Intermittent heteroclinic channel .................... 160

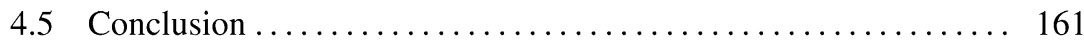


Acknowledgments ................................ 163

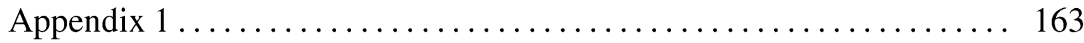

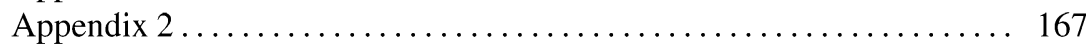

References......................................... 169

\section{Dynamics of Soliton Chains: From Simple to Complex and Chaotic}

\section{Motions}

Konstantin A. Gorshkov, Lev A. Ostrovsky and Yury A. Stepanyants . .. 177

5.1 Introduction .................................. 177

5.2 Stable soliton lattices and a hierarchy of envelope solitons....... 179

5.3 Chains of solitons within the framework of the Gardner model .... 188

5.4 Unstable soliton lattices and stochastisation................ 193

5.5 Soliton stochastisation and strong wave turbulence in a resonator with external sinusoidal pumping .............. 202

5.6 Chains of two-dimensional solitons in positive-dispersion media ........................................ 204

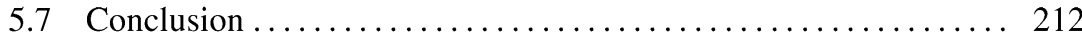

Few words in memory of George M. Zaslavsky .............. 212

References...................................... 214

6 What is Control of Turbulence in Crossed Fields?-Don't Even Think of Eliminating All Vortexes!

Dimitri Volchenkov .................................... $2 \mathbf{2 1 9}$

6.1 Introduction ................................. 220

6.2 Stochastic theory of turbulence in crossed fields: vortexes of all sizes die out, but one ............................ 221

6.2.1 The method of renormalization group .............. 221

6.2.2 Phenomenology of fully developed isotropic turbulence ... 224

6.2.3 Quantum field theory formulation of stochastic

Navier-Stokes turbulence ......................... 226

6.2.4 Analytical properties of Feynman diagrams........... 229

6.2.5 Ultraviolet renormalization and RG-equations .......... 229

6.2.6 What do the RG representations sum?............. 233

6.2.7 Stochastic magnetic hydrodynamics ............... 233

6.2.8 Renormalization group in magnetic hydrodynamics ...... 235

6.2.9 Critical dimensions in magnetic hydrodynamics ......... 238

6.2.10 Critical dimensions of composite operators in magnetic hydrodynamics .......................... 240

6.2.11 Operators of the canonical dimension $d=2 \ldots \ldots \ldots \ldots 241$

6.2.12 Vector operators of the canonical dimension $d=3 \ldots \ldots .242$

6.2.13 Instability in magnetic hydrodynamics .............. 243

6.2.14 Long life to eddies of a preferable size ............. 244

6.3 In search of lost stability . . . . . . . . . . . . . . . . . . . . 249

6.3.1 Phenomenology of long-range turbulent transport in the scrape-off layer (SOL) of thermonuclear reactors......... 249 
6.3.2 Stochastic models of turbulent transport in cross-field

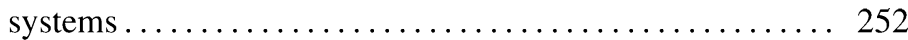

6.3.3 Iterative solutions in crossed fields............. 256

6.3.4 Functional integral formulation of cross-field turbulent

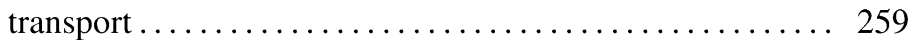

6.3.5 Large-scale instability of iterative solutions .......... 262

6.3.6 Turbulence stabilization by the poloidal electric drift ..... 266

6.3.7 Qualitative discrete time model of anomalous transport

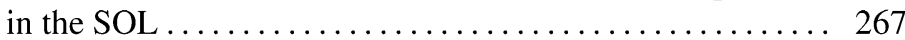

6.4 Conclusion .................................... 272

References....................................... 272

\section{Entropy and Transport in Billiards}

M. Courbage and S.M. Saberi Fathi ...................... 277

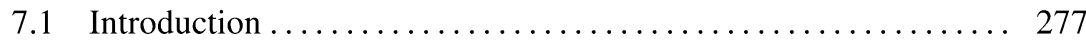

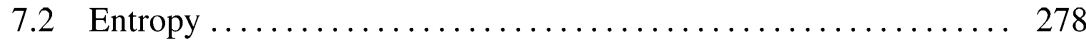

7.2.1 Entropy in the Lorentz gas .................. 283

7.2.2 Some dynamical properties of the barrier billiard model ... 290

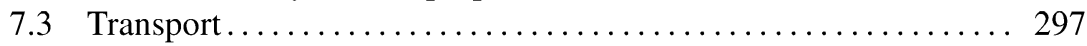

7.3.1 Transport in Lorentz gas........................... 299

7.3.2 Transport in the barrier billiard ................ 301

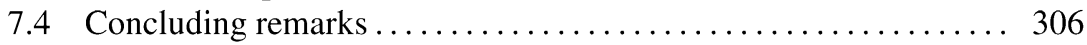

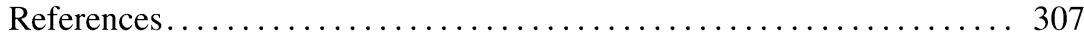

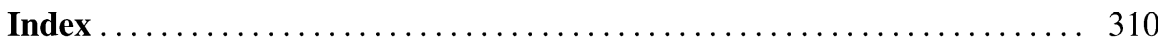

